\title{
Effects of psyllium on LDL-cholesterol concentrations in Brazilian children and adolescents: a randomised, placebo-controlled, parallel clinical trial
}

\author{
Simone Augusta Ribas ${ }^{1 *}$, Diana Barbosa Cunha ${ }^{2}$, Rosely Sichieri ${ }^{2}$ and Luiz Carlos Santana da Silva ${ }^{3}$ \\ ${ }^{1}$ Nutrition Division, State University of Rio de Janeiro, Rio de Janeiro, Brazil \\ ${ }^{2}$ Department of Epidemiology, Social Medicine Institute, State University of Rio de Janeiro, Rio de Janeiro, Brazil \\ ${ }^{3}$ Laboratory of Inborn Errors of Metabolism, Federal University of Pará, Belém, Brazil
}

(Submitted 24 May 2014 - Final revision received 11 September 2014 - Accepted 1 October 2014 - First published online 13 November 2014)

\section{Abstract}

The present study investigated the LDL-cholesterol (LDL-C)-lowering effects of psyllium in Brazilian dyslipidaemic children and adolescents. A total of fifty-one individuals (6-19 years) with mild-to-moderate hypercholesterolaemia were evaluated by conducting a randomised, double-blind, placebo-controlled, parallel clinical trial. Over an 8-week trial period, the participants were randomly allocated to one of two groups (control: $n 25$ and psyllium: $n$ 26) using a computer-generated random number sequence. Fasting blood samples, dietary records and anthropometric data were collected. Both groups were treated with the National Cholesterol Education Program Step 2 diet for 6 weeks before randomisation. After this run-in period, a daily supplement of $7 \cdot 0 \mathrm{~g}$ psyllium was given to the intervention group, while an equivalent amount of cellulose was given to the control group. Statistically significant changes between the control and intervention groups over time were observed for total cholesterol $(7 \cdot 7 \% ;-0.39 \mathrm{mmol} / \mathrm{l} ; P=0.003)$ and LDL-C $(10 \cdot 7 \% ;-0.36 \mathrm{mmol} / \mathrm{l} ; P=0 \cdot 01)$. None of the participants reported any aversion to the smell, taste, appearance or texture of psyllium. No serious adverse effects were reported during the study. In addition to causing a significant reduction in LDL-C concentrations, psyllium therapy was found to be both safe and acceptable for the treatment of hypercholesterolaemic children and adolescents.

\section{Key words: Dyslipidaemia: Psyllium: Children: Adolescents}

Atherosclerotic CVD is a leading cause of morbidity and premature mortality in Western countries and hypercholesterolaemia is recognised as a major cardiovascular risk factor ${ }^{(1)}$. Available data indicate that $28-40 \%$ of Brazilian children and adolescents are dyslipidaemic ${ }^{(2,3)}$.

According to current Brazilian and American guidelines ${ }^{(4,5)}$, the best way to reduce LDL-cholesterol (LDL-C) concentrations is to follow a low-fat diet supplemented with water-soluble fibres and plant stanols and sterols, combined with weight control and exercise ${ }^{(6)}$. Alternative dietary therapies have been proposed and efforts have been made to enhance the efficacy of conventional dietary therapy (low-fat diet) to reduce serum cholesterol concentrations through the inclusion of specific foods or food components with known cholesterol-lowering properties ${ }^{(4,6)}$.

A recent systematic review $^{(7)}$ has demonstrated the cholesterol-lowering capacity of the soluble dietary fibre extracted from psyllium husk in adults. Psyllium has been found to lead to reductions ranging from $2 \cdot 78$ to $-22 \cdot 8 \%$ in the serum LDL-C concentrations of children and adolescents ${ }^{(8-10)}$. The wide range of effects observed in these studies may be related to the quality of the dietary intervention or to the different methods of randomisation, blinding, dietary intake assessment and laboratory measurement.

Psyllium is one of the richest known sources of soluble mucilaginous dietary fibre. It is derived from the seed husk of Plantago ovata ${ }^{(11)}$ and is considered a useful supplement to dietary therapy (National Cholesterol Education Program (NCEP) Step 1 and 2 diets) for the treatment of hypercholesterolaemic patients. Despite this, Haney et al. ${ }^{(12)}$ and Spence et $a l^{(13)}$ have expressed certain doubts with regard to the dose-response relationship and long-term efficacy of the treatment.

Although these positive effects have been clearly demonstrated in hypercholesterolaemic adults ${ }^{(7)}$, there are few, mostly old, published studies on the use of psyllium or other fibre mixtures in children or adolescents ${ }^{(9,10)}$ and some ${ }^{(13,14)}$ have reported inconclusive results.

We hypothesised that psyllium supplementation exerts an additional lipid-lowering effect on serum LDL-C concentrations in dyslipidaemic paediatric subjects treated with the NCEP Step 2 diet.

Abbreviations: HDL-C, HDL-cholesterol; LDL-C, LDL-cholesterol; NCEP, National Cholesterol Education Program; TC, total cholesterol. 
Therefore, in the present study, the safety and efficacy of the use of psyllium for treating hypercholesterolaemic children and adolescents from Brazil were evaluated.

\section{Experimental methods \\ Setting and participants}

Children and adolescents aged between 6 and 19 years who were diagnosed with mild-to-moderate hypercholesterolaemia were recruited to the study. All the participants were outpatients of the Paediatric Nutrition Division (PND) and Adolescent Health Studies Nucleus of the Pedro Ernesto University Hospital (HUPE) in Rio de Janeiro, Brazil, and were recruited between August 2008 and January 2010. The first patient was recruited on 4 August 2008 and the last patient on 20 November 2010. Individuals with a fasting plasma total cholesterol (TC) concentration $>4.40 \mathrm{mmol} / \mathrm{l}$ and initially treated with the PND standard therapeutic low-fat diet, which is similar to the NCEP Step $1 \operatorname{diet}^{(4)}$, for at least 6 weeks were eligible to participate in the study.

Patients with diabetes or other chronic medical conditions were excluded from the study, as were those with CVD and those taking lipid-lowering agents or consuming functional foods. Patients with TAG concentrations $>3.39 \mathrm{mmol} / \mathrm{l}$ or with BMI-for-age $Z$-score greater than +2 were also excluded. All patients were required to maintain a constant body weight ( $\pm 5 \%)$ throughout the study.

\section{Ethical approval}

The present study was conducted according to the guidelines of the Declaration of Helsinki, and all procedures involving human subjects were approved by the Ethics Committee of the HUPE (Rio de Janeiro State University, Brazil). The experimental protocol and informed consent forms were analysed and approved by the HUPE review board in August 1997 (protocol 1772), and written consent was obtained from each adolescent and from the parents/guardians of children after a detailed explanation of the procedure.

\section{Experimental protocol}

The present study was carried out as a randomised, doubleblind, placebo-controlled, parallel clinical trial (Fig. 1) in two phases. It was conducted and reported according to the CONSORT guidelines. It is registered at http://www. ensaiosclinicos.gov.br (Brazilian Clinical Trials Identifier: RBR5fybg2). All ongoing and related trials for this drug/intervention are registered. Trial registration for the study was done after recruitment of the participants due to lack of knowledge regarding the researcher mandatory registration, plus the National Council for Research Ethics, before starting the trial.

After screening, all the patients who were recruited were allowed to adapt to the NCEP Step 2 diet $^{(4)}$ for 6 weeks before the start of the experiment. Blood samples were collected at the beginning of the adaptation phase (week -6) and at the start of the experiment (week 0) for the establishment of baseline serum lipid concentrations.

At the end of the adaptation phase, all patients who fulfilled the eligibility criterion (TC concentrations $>4.40 \mathrm{mmol} / \mathrm{l}$ and LDL-C concentrations $>2.84 \mathrm{mmol} / \mathrm{l}$ ) were required to follow the NCEP Step 2 diet and were randomly allocated to one of two groups (control and psyllium) using a computergenerated random number sequence. The experimental (psyllium) group was supplemented with $7 \cdot 0 \mathrm{~g}$ of psyllium daily (Fig. 1), while the control group was supplemented with an equivalent amount of cellulose (placebo) daily for a further 8 weeks.

The composition of diets followed by the two groups was concealed during the study period; information regarding the composition of diets was maintained in sealed folders at a secure location. Neither the dietitian who scheduled the research nor the laboratory personnel responsible for the analyses were aware of the composition of diets of the two groups.

Pubertal stages $(\mathrm{I}-\mathrm{V})^{(15)}$ as well as physical activity status and family history of dyslipidaemia were determined at the beginning of the experiment (week -6). The weight, height and waist circumference of the participants were measured using a calibrated anthropometric scale (Filizola) with a capacity of $150 \mathrm{~kg}$ accurately to $100 \mathrm{~g}$, a stadiometer (Filizola) accurately to $0 \cdot 1 \mathrm{~cm}$ and an inelastic metric tape with a maximum length of $150 \mathrm{~cm}$ and a precision of $0 \cdot 1 \mathrm{~cm}$, respectively.

Standard anthropometric indices $\left(\mathrm{BMI}\left(\mathrm{kg} / \mathrm{m}^{2}\right)\right.$, waist:hip ratio and body fat percentage) were calculated for each participant at the beginning of the experiment (week -6) and at the end of each dietary treatment period (week 8). BMI values were adjusted for age and sex for each participant based on the 2007 WHO growth charts ${ }^{(16)}$. Skinfold thickness measurements were based on the standard protocol ${ }^{(17)}$, and body fat percentage was estimated using Slaughter's equation $^{(18)}$. The participants were considered to be physically active if they engaged in some physical activity, including light exercise such as walking, for at least $300 \mathrm{~min} /$ week $^{(19)}$.

At each visit during the dietary treatment phase, the participants were asked about possible adverse effects or intolerance to psyllium or placebo. Serum lipid profiles and glucose concentrations were determined at weeks $-6,0,4$ and 8 .

\section{Sample size}

Randomisation order was established before the recruitment of participants, and a minimum sample size of fifty subjects (i.e. twenty-five in each group) as required by the $80 \%$ power calculation was aimed for to detect a change of above $5 \%$ in LDL-C concentrations ${ }^{(9)}$ in the treatment groups with a significance level of $0 \cdot 05$. To carry out the study, twenty-five and twenty-six participants were enrolled into the control and psyllium groups, respectively, during randomisation.

\section{Dietary treatments}

The NCEP Step 2 diet provides $55 \%$ of total energy as carbohydrates, $15 \%$ as protein, $<30 \%$ as fat and $<7 \%$ as SFA, with 


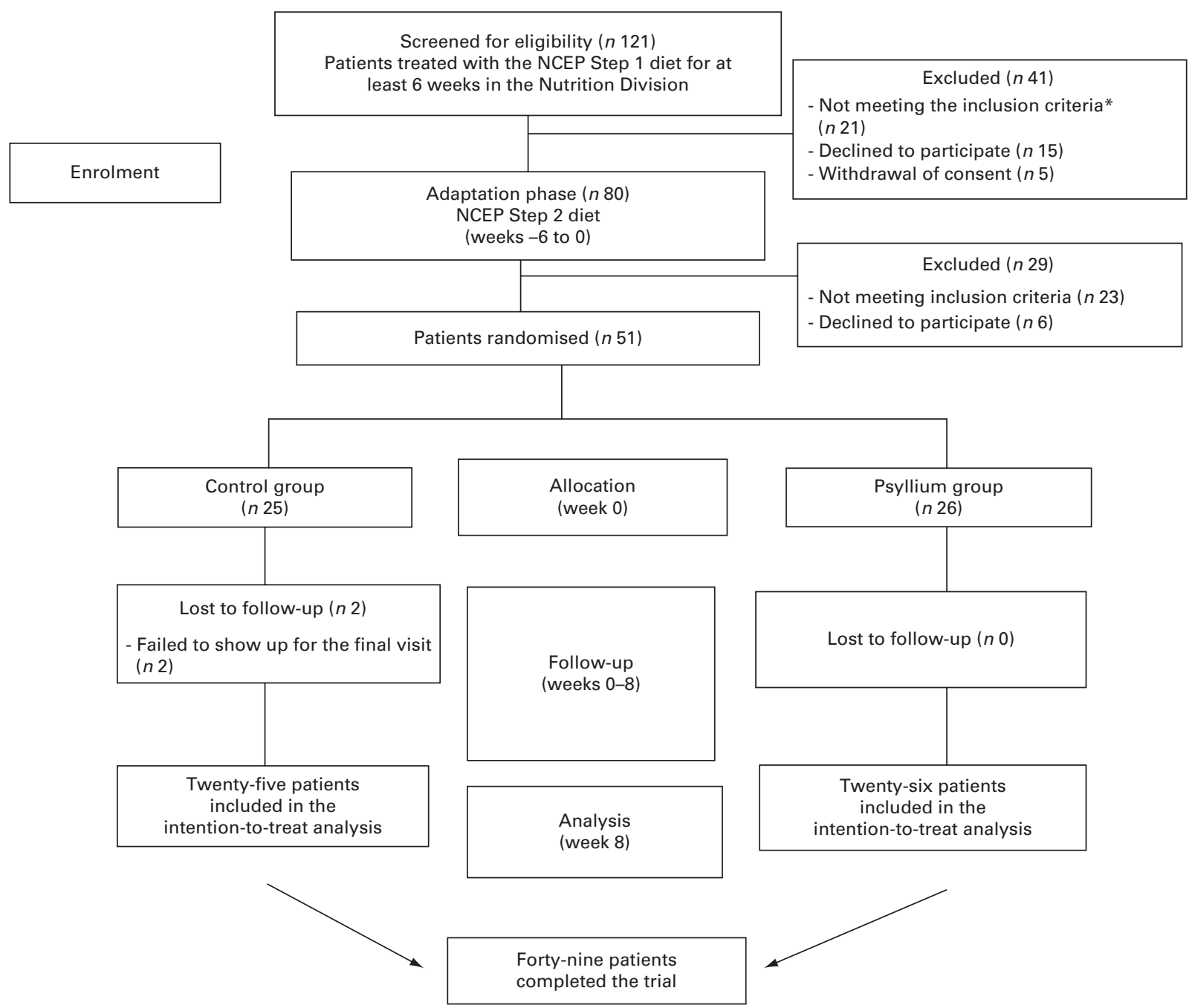

Fig. 1. Flow diagram depicting the progress of participants in the trial. ${ }^{*}$ Total cholesterol concentrations $>4.4 \mathrm{mmol} / \mathrm{l}$. NCEP, National Cholesterol Education Program.

a daily cholesterol intake $<200 \mathrm{mg}$. Qualified dietitians monitored nutrient intake and provided dietary counselling to the participants. The participants were asked to maintain food records for three non-consecutive days, including one weekend day. Dietary compliance and tolerance were evaluated using food frequency records at weeks $-6,0,4$ and 8 and monitored by carrying out interviews with the participants and counting the empty sachets of psyllium. Computerised nutrient analyses were conducted by trained HUPE qualified dietitians using the Avanutri Revolution software package (Avanutri Informática Ltda). Energy derived from total fat, saturated fat, monounsaturated fat, polyunsaturated fat, dietary cholesterol, carbohydrates, total fibre and protein was also evaluated according to the Dietary Reference Intakes established by the US National Academy of Sciences ${ }^{(20)}$.

During the dietary treatment phase, the psyllium group was given orange-flavoured sugar-free psyllium Laxofibra ${ }^{\circledR}$ (Almeida Prado), providing $3.5 \mathrm{~g}$ of hydrophilic mucilloid per $5 \mathrm{~g}$ of powder. The control group was given $3.5 \mathrm{~g}$ of inert microcrystalline cellulose (Avicel PH-101; FMC Corporation) twice daily instead of psyllium. The powders supplied to both groups were similar in colour and texture, and any differences in flavour would have been masked by the taste of the beverage in which the powders were mixed. All the participants were instructed to consume the fibre (psyllium or cellulose) in liquid form (water or juices) in the morning and afternoon.

\section{Laboratory analyses}

Blood samples were analysed by the Laboratory of Clinical Analysis Centre, Rio de Janeiro State University. The participants fasted for $12-14 \mathrm{~h}$ before the collection of blood samples. Fasting plasma glucose concentrations were determined using the oxidase method (Konelab 60i; Wiener $\mathrm{Lab}^{\circledR}$ ). The Konelab enzymatic method was used to measure TC, HDL-cholesterol (HDL-C) and TAG concentrations. LDL-C concentrations were determined from TC, HDL-C, and TAG concentrations using the Friedewald equation ${ }^{(21)}$. The cut-offs used for determining the serum concentrations of TC, TAG and HDL-C were based on the values determined by the $\mathrm{NCEP}^{(4)}$. Details regarding safety and tolerance to 
psyllium were obtained using an open-ended questionnaire referring to any unusual symptoms or discomfort or side effects such as increased defecation, bloating, flatulence or fullness during the treatment period.

\section{Statistical analyses}

An intention-to-treat analysis was conducted to evaluate the variation in TAG, TC and lipid fractions over time according to the allocation group (control/intervention). This analysis was carried out with random regression using the PROC MIXED procedure in SAS. The main effect of psyllium supplementation was determined using a global treatment group $\times$ time interaction term. The level of significance was set at 0.05 . The random intercept $\times$ random intercept slope models, adequate covariance parameter and the possibility of quadratic analysis were tested for each analysis.
The predicted mean values are shown in Fig. 2. All analyses were performed using SAS 9.3 (SAS Institute, Inc.).

\section{Results}

\section{Participant characteristics}

A flow diagram depicting the progress of participants in the trial is shown in Fig. 1. Of the 121 outpatients screened for enrolment in the study, eighty were qualified to participate in the NCEP Step 2 diet (adaptation) phase. After 6 weeks, fifty-one of the eighty participants who began the dietary adaptation phase were qualified for inclusion in the clinical trial (control: $n 25$ and psyllium: $n$ 26) and were included in the intention-to-treat analysis. Disqualification was primarily due to the failure of participants to fulfil the inclusion criteria ( $n$ 23), while a further six participants withdrew consent. Subsequently, two participants were excluded after
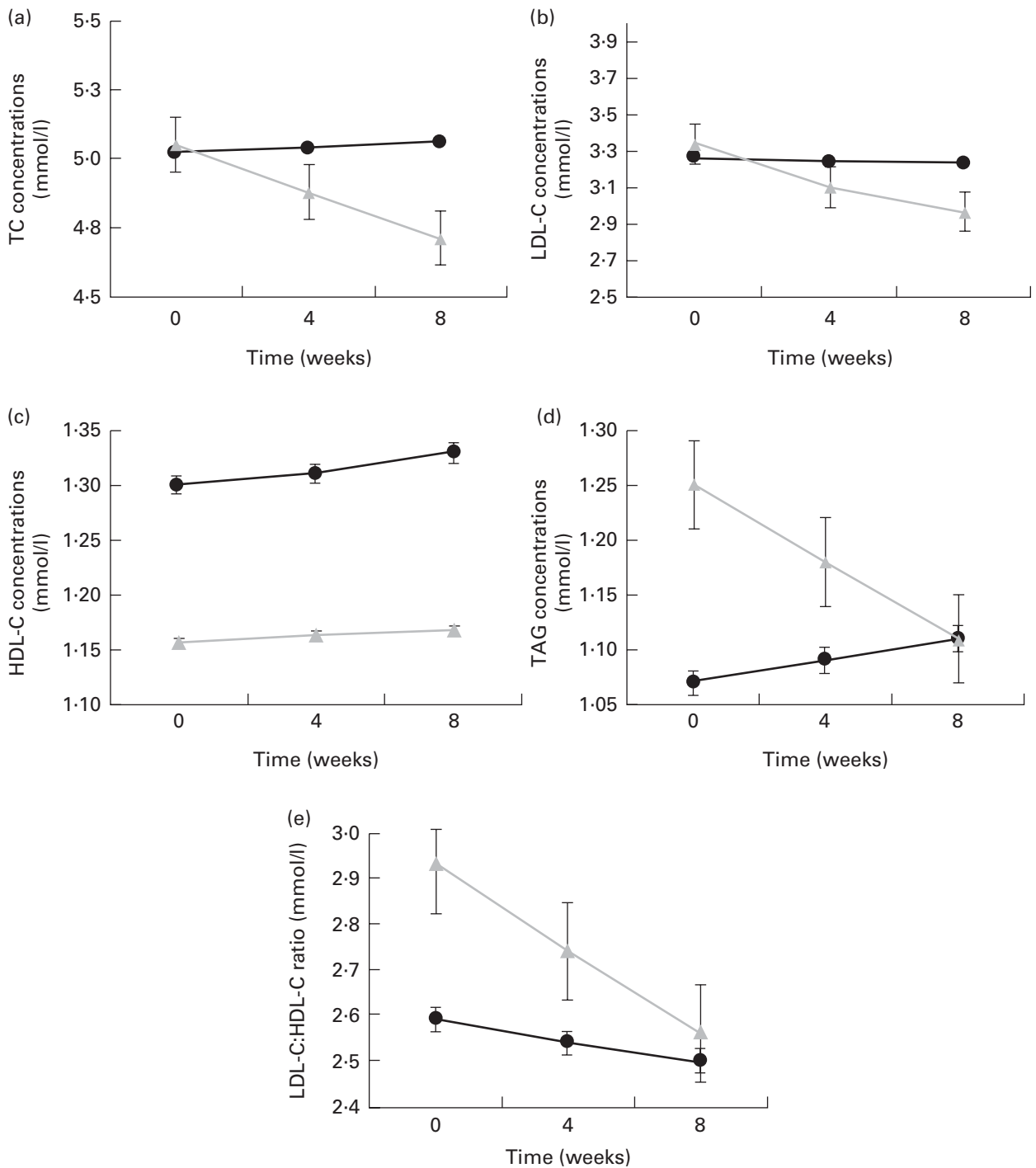

Fig. 2. Changes in serum (a) total cholesterol (TC) concentrations $\left(P_{\text {treatmentxtime }}<0.01\right)$, (b) LDL-cholesterol (LDL-C) concentrations $\left(P_{\text {treatmentxtime }}=0.01\right)$, (c) HDL-cholesterol (HDL-C) concentrations $\left(P_{\text {treatmentxtime }}=0.72\right)$, (d) TAG concentrations $\left(P_{\text {treatmentxtime }}=0.10\right)$ and $(\mathrm{e})$ LDL-C:HDL-C ratio $\left(P_{\text {treatmentx }}\right.$ time $=0.45$ ) at baseline and after 4 and 8 weeks of treatment. $\rightarrow-$, Control group; $\rightarrow-$, psyllium group. 
randomisation, leaving forty-nine participants (control: $n 23$ and psyllium: $n$ 26).

The baseline analysis revealed that more than half of the participants were inactive, two-thirds had a family history of dyslipidaemia, and more than three-quarters were from low-income backgrounds. No significant differences were observed between the control and psyllium groups $(P>0.05)$.

Both the control and psyllium groups had similar anthropometric indices. No significant changes from baseline values were recorded in either group during the clinical visits, and the difference between the baseline and post-treatment body weight values was less than $5 \%$ in both groups (Table 1).

The baseline and post-treatment daily dietary intakes of the two groups are summarised in Table 2 . Detailed analysis revealed no significant differences between the groups for fifteen of the seventeen dietary indices evaluated. The baseline-to-posttreatment intakes of energy $(P=0 \cdot 008)$ and carbohydrates ( $P=0.009)$ decreased significantly in the psyllium group. However, there was no significant correlation between these indices and lipid profiles. Further analysis adjusting for energy intake did not reveal any changes in the results.

The mean cholesterol and saturated fat intakes of the two groups were in accordance with the dietary guidelines for the prevention of dyslipidaemia ${ }^{(3)}$.

\section{Primary outcome}

At baseline, both the control and psyllium groups had similar lipid concentrations and exhibited no significant differences in serum TC, LDL-C or HDL-C, or TAG concentrations.
The effects of dietary treatments on lipid profiles after 8 weeks are summarised in Table 3. Mean TC and LDL-C concentrations were $6.7 \% \quad(-0.34 \mathrm{mmol} / 1, \quad P=0.01)$ and $11.0 \%(-0.37 \mathrm{mmol} / 1, P=0.02)$ lower than baseline values, respectively, and $7.7 \%(-0.39 \mathrm{mmol} / 1, P=0.01)$ and $10.7 \%$ $(-0.36 \mathrm{mmol} / 1, P=0.01)$ from placebo. Significant changes were also observed in the lipid profiles of the psyllium group when compared with those of the control group (TC: $\quad 7.7 \% \quad(-0.39 \mathrm{mmol} / \mathrm{l}), \quad P=0.003) ; \quad$ LDL-C: $\quad 10.7 \%$ $(-0.36 \mathrm{mmol} / \mathrm{l}), \quad P=0.01)$ during the 8 -week treatment period (Fig. 2 (a) and (b)).

Psyllium treatment had no significant effect on TAG concentrations, HDL-C concentrations and LDL-C:HDL-C ratio (Table 3 and Fig. 2 (c)-(e)).

During the 8 -week treatment period, normal cholesterol values $(\leq 4.40 \mathrm{mmol} / \mathrm{l})$ were achieved in six $(23.0 \%)$ of the twenty-six participants in the psyllium group $v$. one (4\%) of the twenty-five participants in the control group.

\section{Secondary outcome}

The mean total and saturated fat and cholesterol intakes of the participants (26.6 (SD 51), $6.0(\mathrm{SD} 2 \cdot 3)$ and $148.0(\mathrm{SD} 44) \mathrm{mg} / \mathrm{d})$ before and after the treatment were in accordance with the guidelines for the prevention of dyslipidaemia. In contrast, the average dietary fibre intake was $16 \cdot 8$ (SD 5.1) g/d, which is below the nutritional recommendations for teenagers ${ }^{(20)}$.

Dietary compliance was excellent overall (94\% in the control group and $91 \%$ in the psyllium group), and there were no significant differences between the groups.

Table 1. Baseline characteristics of the participants (Number of participants and percentages; mean values and $95 \%$ confidence intervals)

\begin{tabular}{|c|c|c|c|c|}
\hline \multirow[b]{2}{*}{ Variables } & \multicolumn{2}{|c|}{ Control group $(n 25)$} & \multicolumn{2}{|c|}{ Psyllium group $(n 26)$} \\
\hline & $n$ & $\%$ & $n$ & $\%$ \\
\hline Males & 13 & $52 \cdot 0$ & 13 & $50 \cdot 0$ \\
\hline Females & 12 & $48 \cdot 0$ & 13 & $50 \cdot 0$ \\
\hline Children & 10 & $40 \cdot 0$ & 11 & $42 \cdot 3$ \\
\hline Adolescents & 15 & $60 \cdot 0$ & 15 & $57 \cdot 7$ \\
\hline \multicolumn{5}{|l|}{ Tanner stages of sexual maturity } \\
\hline I (pre-puberty) & 8 & $32 \cdot 0$ & 6 & $23 \cdot 1$ \\
\hline II-III (puberty) & 14 & $56 \cdot 0$ & 17 & 65.4 \\
\hline IV-V (post-puberty) & 3 & $12 \cdot 0$ & 3 & 11.5 \\
\hline Inactivity & 15 & $60 \cdot 0$ & 18 & $69 \cdot 2$ \\
\hline \multirow[t]{2}{*}{ Positive family history of dyslipidaemia } & 16 & $64 \cdot 0$ & 18 & $69 \cdot 2$ \\
\hline & Mean & $95 \% \mathrm{Cl}$ & Mean & $95 \% \mathrm{Cl}$ \\
\hline \multicolumn{5}{|l|}{ Anthropometry } \\
\hline BMI $\left(\mathrm{kg} / \mathrm{m}^{2}\right)$ & 21.65 & $20 \cdot 0,23 \cdot 2$ & 21.87 & $20 \cdot 3,23 \cdot 4$ \\
\hline Body fat percentage & $28 \cdot 3$ & $24 \cdot 0,32 \cdot 3$ & $31 \cdot 1$ & $27 \cdot 0,35 \cdot 4$ \\
\hline Waist circumference $(\mathrm{cm})$ & $77 \cdot 1$ & $72 \cdot 6,82 \cdot 8$ & $77 \cdot 0$ & $72 \cdot 1,82 \cdot 1$ \\
\hline Waist:hip ratio & 0.86 & $0.84,0.90$ & 0.87 & $0.84,0.91$ \\
\hline \multicolumn{5}{|l|}{ Biochemistry } \\
\hline Total cholesterol $(\mathrm{mmol} / \mathrm{l})$ & 5.04 & $4.83,5.24$ & 5.04 & $4 \cdot 82,5 \cdot 26$ \\
\hline LDL-C (mmol/l) & $3 \cdot 28$ & $3.02,3.54$ & 3.37 & $3.15,3.59$ \\
\hline $\mathrm{HDL}-\mathrm{C}(\mathrm{mmol} / \mathrm{l})$ & 1.31 & $1.22,1.41$ & $1 \cdot 16$ & $1.09,1.23$ \\
\hline LDL-C:HDL-C ratio & 2.56 & $2 \cdot 28,2 \cdot 86$ & 2.95 & $2 \cdot 73,3 \cdot 2$ \\
\hline $\mathrm{TAG}(\mathrm{mmol} / \mathrm{l})$ & 1.01 & $0.88,1.15$ & $1 \cdot 27$ & $0.99,1.55$ \\
\hline
\end{tabular}

LDL-C, LDL-cholesterol; HDL-C, HDL-cholesterol. 
Table 2. Baseline and post-treatment (after 8 weeks) daily dietary intakes of the participants (Mean values and standard deviations)

\begin{tabular}{|c|c|c|c|c|c|c|c|c|}
\hline & \multicolumn{4}{|c|}{ Control group (n 25) } & \multicolumn{4}{|c|}{ Psyllium group (n 26) } \\
\hline & \multicolumn{2}{|c|}{ Baseline } & \multicolumn{2}{|c|}{ Post-treatment } & \multicolumn{2}{|c|}{ Baseline } & \multicolumn{2}{|c|}{ Post-treatment } \\
\hline & Mean & SD & Mean & SD & Mean & SD & Mean & SD \\
\hline Energy (kJ) & 7757 & 1475 & 7380 & 1421 & 7819 & 1713 & $6919^{*}$ & 1306 \\
\hline Total fat $(\mathrm{g})$ & $57 \cdot 0$ & $16 \cdot 5$ & $57 \cdot 0$ & $13 \cdot 4$ & $58 \cdot 7$ & $15 \cdot 3$ & $54 \cdot 8$ & $12 \cdot 6$ \\
\hline Percentage of energy & $26 \cdot 3$ & $5 \cdot 2$ & $27 \cdot 8$ & $4 \cdot 3$ & $27 \cdot 1$ & 5.5 & 28.4 & $5 \cdot 3$ \\
\hline $\operatorname{SFA}(g)$ & $13 \cdot 5$ & $7 \cdot 4$ & 13.9 & $6 \cdot 8$ & $12 \cdot 6$ & $5 \cdot 5$ & $12 \cdot 50$ & $4 \cdot 4$ \\
\hline Percentage of energy & $6 \cdot 4$ & $2 \cdot 8$ & $6 \cdot 8$ & $2 \cdot 5$ & $5 \cdot 8$ & $2 \cdot 0$ & $6 \cdot 3$ & $2 \cdot 2$ \\
\hline $\operatorname{MUFA}(\mathrm{g})$ & 14.5 & $6 \cdot 0$ & $14 \cdot 7$ & $6 \cdot 1$ & 14.9 & $5 \cdot 8$ & 14.4 & $5 \cdot 0$ \\
\hline Percentage of energy & $6 \cdot 6$ & $2 \cdot 3$ & $7 \cdot 1$ & $2 \cdot 7$ & 6.9 & 2.6 & $7 \cdot 6$ & 2.5 \\
\hline PUFA $(g)$ & $12 \cdot 0$ & $4 \cdot 9$ & $11 \cdot 8$ & $5 \cdot 1$ & $12 \cdot 2$ & $5 \cdot 3$ & $12 \cdot 2$ & $6 \cdot 0$ \\
\hline Percentage of energy & $5 \cdot 6$ & $2 \cdot 0$ & 5.9 & $2 \cdot 5$ & 5.9 & 2.9 & $6 \cdot 4$ & $3 \cdot 0$ \\
\hline$P: S$ & $1 \cdot 0$ & 0.6 & 0.9 & 0.4 & $1 \cdot 1$ & 0.7 & $1 \cdot 1$ & 0.6 \\
\hline Cholesterol (mg) & $146 \cdot 7$ & $45 \cdot 2$ & $147 \cdot 2$ & 44.9 & $151 \cdot 5$ & $44 \cdot 3$ & $146 \cdot 6$ & $41 \cdot 3$ \\
\hline Protein $(\mathrm{g})$ & 76.5 & $16 \cdot 8$ & $73 \cdot 2$ & $14 \cdot 6$ & $79 \cdot 4$ & $23 \cdot 0$ & 73.4 & $18 \cdot 7$ \\
\hline Percentage of energy & $15 \cdot 9$ & $3 \cdot 3$ & $16 \cdot 0$ & $2 \cdot 8$ & $16 \cdot 3$ & 3.7 & $16 \cdot 8$ & $4 \cdot 2$ \\
\hline Carbohydrates $(\mathrm{g})$ & $249 \cdot 2$ & $57 \cdot 8$ & 231.5 & $49 \cdot 4$ & $251 \cdot 8$ & $65 \cdot 1$ & $217 \cdot 4 \dagger$ & $52 \cdot 6$ \\
\hline Percentage of energy & 51.4 & $8 \cdot 3$ & $49 \cdot 9$ & $5 \cdot 7$ & $51 \cdot 1$ & $5 \cdot 8$ & $50 \cdot 3$ & $5 \cdot 7$ \\
\hline Dietary fibre $(\mathrm{g})$ & $16 \cdot 6$ & 4.4 & $16 \cdot 9$ & $5 \cdot 9$ & $16 \cdot 9$ & $4 \cdot 3$ & $16 \cdot 5$ & $5 \cdot 5$ \\
\hline
\end{tabular}

$\mathrm{P}: \mathrm{S}$, polyunsaturated:saturated fat ratio.

${ }^{*}$ Mean value was significantly different from that of the control group $(P=0.008)$.

† Mean value was significantly different from that of the control group $(P=0.009)$.

In general, psyllium was well tolerated. No serious adverse effects were reported during the study, and only one child complained of gastrointestinal problems with slight abdominal bloating.

\section{Discussion}

The present study is the first randomised, double-blind, placebo-controlled, parallel clinical trial to test the use of psyllium as a supplement to the NCEP Step 2 diet in Brazilian children. Although there is difficulty in obtaining a representative sample of hypercholesterolaemic children for an interventional study, especially in Brazil, all the published studies on this subject have used sample sizes that are similar to or smaller than that used in the present study.

Most of the randomised controlled trials that have investigated the effect of psyllium on cholesterol concentrations in children $^{(9)}$ have used the NCEP Step 1 diet $^{(4)}$. In the present study, by contrast, this diet was used for assessing the eligibility of participants and only those with LDL-C concentrations $>2.4 \mathrm{mmol} / \mathrm{l}$ after treatment with the NCEP Step 1 diet were included in the trial, in which the NCEP Step 2 diet was used. Thus, additional subtle changes with respect to diet would be unlikely.

The addition of supplemental soluble fibre (psyllium) to the NCEP Step 1 diet may lead to further lowering of LDL-C concentrations by $10-15 \%$. However, in children consuming the more stringent Step 2 diet, the addition of water-soluble fibre may have less additional effects on LDL-C concentrations $^{(22)}$. As recommended by the NCEP Expert Panel on Blood Cholesterol Levels in Children and Adolescents, dietary therapy, i.e. a diet low in total fat, saturated fat and cholesterol, remains the cornerstone for treating children and adolescents with elevated LDL-C concentrations ${ }^{(4)}$.

Previous studies have also indicated that this type of fibre has no effect on HDL-C or TAG concentrations when included in a diet low in saturated fat ${ }^{(9,10)}$. In accordance with the literature, the results of the present study showed that the

Table 3. Changes in the lipid profiles of participants from baseline to the end of the dietary treatment period $\left(8\right.$ weeks) ${ }^{*}$

(Mean differences and $95 \%$ confidence intervals)

\begin{tabular}{|c|c|c|c|c|}
\hline \multirow[b]{2}{*}{ Variables } & \multicolumn{2}{|c|}{ Control group ( $n$ 25) } & \multicolumn{2}{|c|}{ Psyllium group $(n 26)$} \\
\hline & Mean & $95 \% \mathrm{Cl}$ & Mean & $95 \% \mathrm{Cl}$ \\
\hline Total cholesterol $(\mathrm{mmol} / \mathrm{l})$ & 0.05 & $-0.09,0.19$ & -0.34 & $-0.51,-0.17$ \\
\hline LDL-C (mmol/l) & -0.01 & $-0.15,0.14$ & -0.37 & $-0.53,-0.21$ \\
\hline $\mathrm{HDL}-\mathrm{C}(\mathrm{mmol} / \mathrm{l})$ & 0.03 & $-0.05,0.10$ & 0.01 & $-0.06,0.08$ \\
\hline LDL-C:HDL-C ratio & 0.00 & $-0.01,0.01$ & -0.01 & $-0.02,0.00$ \\
\hline $\mathrm{TAG}(\mathrm{mmol} / \mathrm{l})$ & 0.02 & $-0.12,0.15$ & -0.15 & $-0.27,-0.03$ \\
\hline
\end{tabular}

LDL-C, LDL-cholesterol; HDL-C, HDL-cholesterol.

${ }^{*}$ Results from mixed models that include treatment, time and time $\times$ treatment variables. 
consumption of psyllium in conjunction with the NCEP Step 2 diet helps to maintain lower LDL-C concentrations without affecting HDL-C or TAG concentrations ${ }^{(7,10)}$.

The $11 \%(-0.37 \mathrm{mmol} / \mathrm{l})$ reduction in LDL-C concentrations observed in the present study is consistent with the results of previous psyllium trials in adults ${ }^{(7)}$, but higher than that recorded in short-term studies carried out previously in children and adolescents ${ }^{(9,23)}$. Although this change may be relatively small in comparison with that generated by pharmacological agents, interventions producing even a small reduction in LDL-C concentrations may have important implications for public health standards if applied to a sufficient number of people and maintained over the long term.

The results of the present study also revealed that normal cholesterol values were achieved in six (23.0\%) participants in the psyllium group, suggesting that psyllium could replace the lipid-lowering medication in cases of mild hypercholesterolaemia $(4.40-4.65 \mathrm{mmol} / \mathrm{l})$. Furthermore, it is important to remember that because of possible side effects, lipid-lowering drugs are not recommended for children, demonstrating the importance of including lipid-lowering functional foods in the diet of dyslipidaemic patients.

Dietary therapy is generally a relatively inexpensive and cost-effective approach to the primary prevention of coronary artery disease in high-risk individuals ${ }^{(4,23)}$, and low-cost, welltolerated supplements to the NCEP diets, such as palatable foods containing psyllium, appear to be potentially valuable resources for the long-term maintenance of low LDL-C concentrations using non-pharmacological therapies ${ }^{(24)}$.

Despite the beneficial effects observed in the present study, determination of the adequacy of dietary fibre interventions in the Brazilian population, especially in younger individuals, is a difficult task for nutritionists. The results of the present study also showed that adolescents consumed less vegetables and fruits and more refined foods despite the dietary guidelines provided, which in consequence contributed to the average dietary fibre intake being below the nutritional recommendations in this age group ${ }^{(20,25)}$.

The participants of the present study exhibited excellent compliance and good dietary tolerance (96\%), which indicates that psyllium is a palatable source of water-soluble fibre. In this case, the inclusion of psyllium in the diet may be a potentially useful mechanism for the treatment of hypercholesterolaemic children who are unwilling to take medication or unable to tolerate medication (bile acid resins or statins) or are too young for such treatment.

Although other sources of fibre such as $\beta$-glucan, guar gum and pectin ${ }^{(26)}$ may also help to reduce TC and LDL-C concentrations, their use is restricted by their lack of palatability ${ }^{(27)}$. Conversely, psyllium is one of the most widely used fibre supplements because it is reasonably cheap and is better tolerated than other fibre supplements ${ }^{(28)}$. In the present study, none of the participants reported any aversion to the smell, taste, appearance or texture of psyllium, which allowed its effective incorporation into the therapeutic diet. Psyllium can be easily incorporated into foods such as bread, breakfast cereals and pastas and may thus be acceptable as part of a chronic therapy programme for the section of population that suffers from genetic lipoprotein disorders ${ }^{(29)}$.

The study findings indicate that psyllium may be a viable dietary treatment alternative for the prevention of dyslipidaemia in children; therefore, more scientific research must be carried out to verify its effectiveness in the long term.

The results of the present study indicate that psyllium can exert significant short-term (8 weeks) beneficial effects on serum TC and LDL-C concentrations in children when combined with a low-fat diet. The results also indicate psyllium therapy to be a safe, acceptable and effective complement to dietary therapy for the attenuation of lipid risk factors in paediatric CVD patients.

\section{Acknowledgements}

The authors thank dietitians Denise Dutra Weiner and Simone L. Jardim for their technical assistance.

The present study was supported by the Almeida Prado Pharmaceutical Laboratory.

The authors' contributions are as follows: S. A. R. was responsible for the conception and design of the study, acquisition of the data, and analysis and interpretation of the data and contributed to the drafting of the manuscript; D. B. C. performed the data analysis; R. S. and L. C. S. d. S. critically revised the manuscript for important intellectual content and approved the final version to be published.

None of the authors has any conflicts of interest, financial or otherwise, to declare.

\section{References}

1. Guardamagna O, Baracco V, Abello F, et al. (2009) Identification and management of dyslipidemic children. Minerva Pediatr 61, 391-398.

2. Lima SCV, Lyra CO, Pinheiro LGB, et al. (2011) Association between dyslipidemia and anthropometric indicators in adolescents. Nutr Hosp 26, 304-310.

3. Ribas SA \& Silva LCS (2009) Dyslipidemia in schoolchildren from private schools in Belém. Arq Bras Cardiol 292, 446-451.

4. National Cholesterol Education Program (1992) Report of the expert panel on blood cholesterol levels in children and adolescents. Pediatrics 89, Suppl. 1, 525-584.

5. Giuliano ICB, Caramelli B, Pellanda L, et al. (2005) I Diretriz Brasileira para a prevenção da aterosclerose na infância e na adolescência (Guideline for preventing atherosclerosis in childhood and adolescence). Arq Bras Cardiol 85, Suppl. $1, \mathrm{~S} 4-\mathrm{S} 36$

6. Kwiterovich PO (2008) Recognition and management of dyslipidemia in children and adolescents. J Clin Endocrinol Metab 93, 4200-4209.

7. Wei ZH, Wang $\mathrm{H}$, Chen XY, et al. (2009) Time- and dose-dependent effect of psyllium on serum lipids in mildto-moderate hypercholesterolemia: a meta-analysis of controlled clinical trials. Eur J Clin Nutr 63, 821-827.

8. Williams CL, Bollella M, Spark A, et al. (1995) Soluble fiber enhances the hypocholesterolemic effect of the step I diet in childhood. J Am Coll Nutr 14, 251-257. 
9. Moreno LA, Tresaco B, Bueno G, et al. (2003) Psyllium fibre and the metabolic control of obese children and adolescents. J Physiol Biochem 59, 235-242.

10. de Bock M, Derraik JG, Brennan CM, et al. (2012) Psyllium supplementation in adolescents improves fat distribution \& lipid profile: a randomized, participant-blinded, placebocontrolled, crossover trial. PLOS ONE 7, e41735.

11. Singh B (2007) Psyllium as therapeutic and drug delivery agent. Int J Pharm 334, 1-14.

12. Haney E, Huffman L, Bougatsos C, et al. (2007) Screening and treatment for hypercholesterolemia in children and adolescents: systematic evidence review for the U.S. preventive services task force. Pediatrics 120, e180-e214.

13. Spence JD, Huff MW, Heidenheim P, et al. (1995) Combination therapy with colestipol and psyllium mucilloid in patients with hyperlipidemia. Ann Intern Med 123, 493-499.

14. Van Rosendaal GMA, Shaffer EA, Edwards AL, et al. (2004) Effect of time of administration on cholesterol-lowering by psyllium: a randomized cross-over study in normocholesterolemic or slightly hypercholesterolemic subjects. Nutr J 3 , 17.

15. Malina RM \& Bouchard C (1991) Growth, Maturation, and Physical Activity, 2nd ed. Champaign, IL: Human Kinetics Books.

16. World Health Organization (2007) WHO Child Growth Standards: Length/Height-for-Age, Weight-for-Age, Weightfor-Length, Weight-for-Height and Body Mass Indexfor-Age. Methods and Development. Nonserial Publication. Geneva: WHO

17. Lohman TG, Roche A \& Martorell R (1988) Anthropometric Standardization Reference Manual. Champaign, IL: Human Kinetics Books.

18. Slaughter MH, Lohman TG, Boileau RA, et al. (1998) Skinfold equations for estimation of body fatness in children and youth. Hum Biol 60, 709-723.
19. Pate RR, Freedson PS, Sallis JF, et al. (2002) Compliance with physical activity guidelines: prevalence in a population of children and youth. Ann Epidemiol 12, 303-308.

20. Institute of Medicine (2000) Dietary Reference Intakes: Applications in Dietary Assessment. Washington, DC: National Academy Press pp. 306.

21. Friedewald WT, Levy RI \& Fredrickson DS (1972) Estimation of the concentration of low-density lipoprotein cholesterol in plasma, without use of the preparative ultracentrifuge. Clin Chem 18, 499-502.

22. Kwiterovich PO (1995) The role of fiber in the treatment of hypercholesterolemia in children and adolescents. Pediatrics 96, 1005-1009.

23. Solà R, Godàs G, Ribalta J, et al. (2007) Effects of soluble fiber (Plantago ovata husk) on plasma lipids, lipoproteins, and apolipoproteins in men with ischemic heart disease. Am J Clin Nutr 85, 1157-1163.

24. Kinosian BP \& Eisenberg JM (1988) Cutting into cholesterol cost-effective alternatives for treating hypercholesterolemia. JAMA 259, 2249-2254

25. de Moura SA, Pereira RA, Yokoo EM, et al. (2013) Most consumed foods in Brazil: National Dietary Survey 2008-2009. Rev Saúde Pública 47, Suppl. 1, 190S-199S.

26. Theuwissen E \& Mensink RP (2008) Water-soluble dietary fibers and cardiovascular disease. Physiol Behav $\mathbf{9 4}$ 285-292.

27. Anderson JW, Riddell-Mason S, Gustafson NJ, et al. (1992) Cholesterol-lowering effects of psyllium-enriched cereal as an adjunct to a prudent diet in the treatment of mild to moderate hypercholesterolemia. Am J Clin Nutr 56, 93-98.

28. Pal S \& Radavelli-Bagatini S (2012) Effects of psyllium on metabolic syndrome risk factors. Obes Rev 13, 1034-1047.

29. Davidson MH, Dugan LD, Burns JH, et al. (1996) A psylliumenriched cereal for the treatment of hypercholesterolemia in children: a controlled, double-blind, crossover study. $\mathrm{Am} \mathrm{J}$ Clin Nutr 63, 96-102. 